\title{
Gentamicin Sulfate
}

National Cancer Institute

\section{Source}

National Cancer Institute. Gentamicin Sulfate. NCI Thesaurus. Code C65803.

The sulfate salt form of gentamycin, a broad-spectrum aminoglycoside antibiotic complex produced by the fermentation of Micromonospora purpurea or M. echinospora, with antibacterial activity. Gentamicin is a thermostable complex containing the gentamicins C1, C1a, C2, C2a and C2b. 